\title{
Coupling Efficiency Control of Vertically Coupled Microring Resonator Filter by Microactuator
}

\author{
Go Sekiguchi, Naoki Kobayashi, and Yasuo Kokubun, Senior Member, IEEE
}

\begin{abstract}
Coupling efficiency control of vertically coupled microring resonator was successfully realized by microactuator, which is suitable for the simultaneous integration of microelectromechanical systems switch and thermooptic tunable filter. The extinction ratio of drop port response of $15.1 \mathrm{~dB}$, through port response of $2.1 \mathrm{~dB}$, and switching time of $60 \mu \mathrm{s}$ were demonstrated at the applied voltage of $350 \mathrm{~V}$. The loss of through port response in the OFF-state was reduced to $0.8 \mathrm{~dB}$.
\end{abstract}

Index Terms-Coupling efficiency, high index contrast, microactuator, microring, optical waveguide, wavelength switch.

\section{INTRODUCTION}

$\mathbf{M}$ ICRORING resonator filters using high index contrast (HIC) optical waveguides have been recently attracting attention as an add-drop filter for new-generation photonic networks owing to their compactness, functionality such as dispersion compensation, and ease of filter synthesis [1], [2]. To apply the microring resonator add-drop filter to flexible and scalable photonic networks, a tunable microring resonator filter is needed. The authors have proposed and demonstrated a vertically coupled microring resonator filter [3] and a tunable microring filter using polymer materials [4], [5] utilizing the large thermooptic (TO) effect of polymer materials. However, these tunable filters block other wavelength channels during the tuning.

To solve this problem, a hitless wavelength selective switch (WSS) was realized by the series coupled tunable microring resonator filter [6], [7]. Although the hitless switching characteristics were successfully demonstrated in the drop port response, small loss in the through port response has remained in the OFF state. To realize the perfect hitless WSS, we need to reduce the coupling efficiency between the busline and ring waveguides in the OFF state, while keeping an optimum coupling efficiency in the ON state. Therefore, in this study, we introduced a microelectromechanical systems (MEMS)-actuated beam structure to the ring waveguide layer to control the coupling efficiency between the ring and busline waveguides.

\section{Device Structure AND PRINCIPLE}

Fig. 1 shows the structure of MEMS-actuated vertically coupled microring resonator filter. The ring waveguide layer is fab-

Manuscript received March 30, 2006; revised July 31, 2006. This work was supported in part by a Grant-in-Aid for Priority Areas 17068009 from the Ministry of Education, Culture, Sports, Science and Technology, and the 21st Century COE Program in Ministry of Education, Culture, Sports, Science and Technology.

The authors are with the Graduate School of Engineering, Department of Electrical and Computer Engineering, Yokohama National University, Yokohama 240-8501, Japan (e-mail: ykokubun@ynu.ac.jp).

Digital Object Identifier 10.1109/LPT.2006.883167

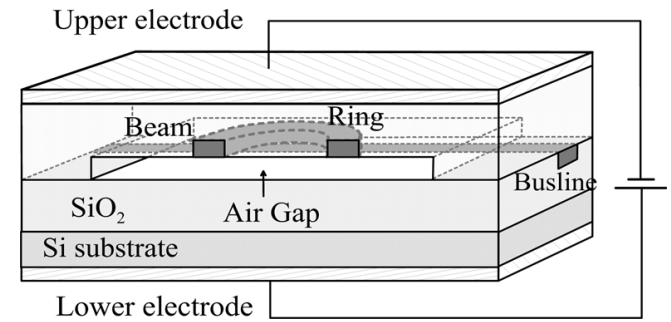

Fig. 1. MEMS-actuated vertically coupled microring resonator filter.

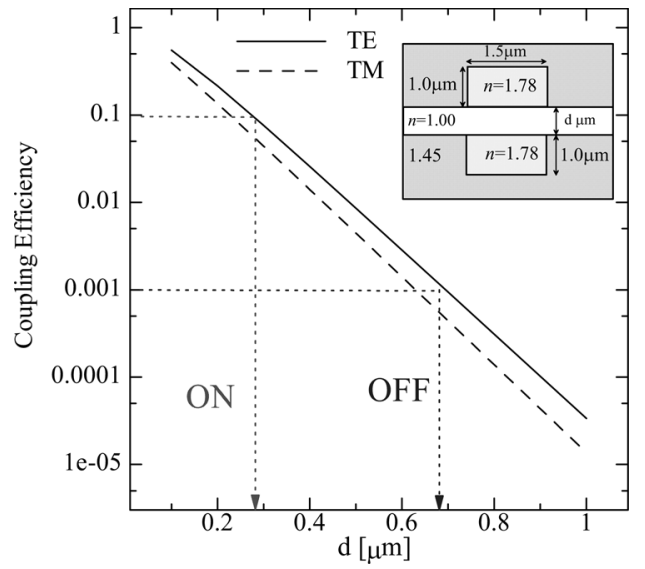

Fig. 2. Calculated coupled efficiency versus air gap between busline and ring waveguide.

ricated inside a straddle mounted beam structure. In this structure, the buffer layer between busline and microring is air and the ring waveguide can be moved by electrostatic force between the upper and lower electrodes, which were formed on the beam and on the reverse side of silicon wafer. The length and width of a beam was designed to be $100-200 \mu \mathrm{m}$ and $50-80 \mu \mathrm{m}$, respectively. The thickness was $3.0-3.5 \mu \mathrm{m}$.

The coupling efficiency of optical power is an important parameter for vertically coupled microring resonator filter. The relation between the coupling efficiency and the gap distance was analyzed using a finite-element mode solver (APSS by Apollo Inc.). The calculated results based on the assumed cross-sectional structure are shown in Fig. 2. To obtain a desirable drop port response with low loss in the ON state, the coupling efficiency should be around 0.1 [8]. It is seen from Fig. 2 that this value approximately corresponds to the air gap of $0.3 \mu \mathrm{m}$ between the busline and ring waveguides. To achieve an extinction ratio of $30 \mathrm{~dB}$ in the OFF-state, the coupling efficiency should be around 0.001 , which approximately corresponds to the air gap of $0.6 \sim 0.7 \mu \mathrm{m}$. Therefore, we should control the air gap in the range of $0.3-0.7 \mu \mathrm{m}$. 


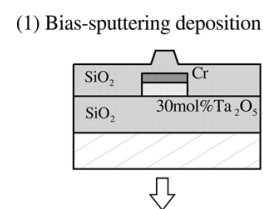

(2) Lift-off of $\mathrm{SiO}_{2}$

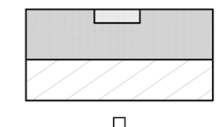

乃

(3) Evaporation of $\mathrm{Al}$, Sputtering depositon of $30 \mathrm{~mol}^{2} \mathrm{Ta}_{2} \mathrm{O}_{5}-\mathrm{SiO}_{2}$

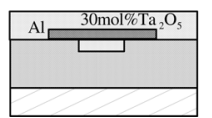

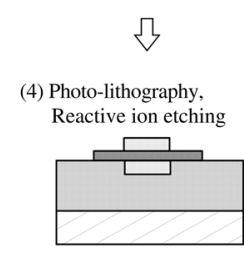

そ)

(5) Chemical vapor deposition of $\mathrm{SiO}_{2}$

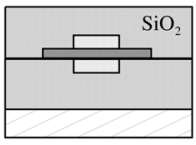

Fig. 3. Fabrication process.

The deflection of beam $D$ increases in proportion to the fourth power of beam length and is inversely proportional to the third power of beam thickness, as expressed by

$$
D=\frac{P l^{4}}{32 E t^{3}}
$$

where $P$ is the uniformly distributed load (electrostatic force), $l$ is the beam length, $E$ is the Young's modulus, and $t$ is the beam thickness. On the other hand, the electrostatic force $P$ is expressed by

$$
P=\frac{V^{2}}{2} \frac{\epsilon_{0}}{\left.\left\{\frac{\epsilon_{0}}{\epsilon}\left(t+t_{\text {clad }}\right)+t_{\text {air }}\right)\right\}^{2}}
$$

where $V$ is the applied voltage, $\epsilon$ is the dielectric constant of the $\mathrm{SiO}_{2}, \epsilon_{0}$ is the dielectric constant of the vacuum, $t_{\text {clad }}$ is the lower cladding thickness, and $t_{\text {air }}$ is the gap length. From these equations, the applied voltage required to obtain the deflection of $0.25 \mu \mathrm{m}$ was evaluated to be $350 \mathrm{~V}$ for a $110-\mu \mathrm{m}$-long beam.

\section{FABRICATION}

The fabrication process is shown in Fig. 3. The busline waveguide and ring core made of $\mathrm{Ta}_{2} \mathrm{O}_{5}-\mathrm{SiO}_{2}(n=1.785$ at $\lambda=1550 \mathrm{~nm}$ ) were formed by RF sputtering deposition technique. A 3-in $\phi \mathrm{Si}$ wafer was used as the substrate and a $5-\mu$ m-thick thermally grown $\mathrm{SiO}_{2}$ was used as the lower cladding. The width and thickness of busline waveguide were 1.5 and $0.9 \mu \mathrm{m}$, respectively, and those of ring waveguide were 1.5 and $1.0 \mu \mathrm{m}$, respectively. The ring radius was $20 \mu \mathrm{m}$. The bending loss of $20-\mu \mathrm{m}$ ring radius was calculated to be less than $0.01 \mathrm{~dB} /$ round. The buried structure was fabricated by the liftoff process [9].

The ring waveguide was buried inside the straddle-mounted beam, which was formed by etching the sacrifice layer after the deposition of the upper cladding. A $\ell$ evaporated film was used as the sacrifice layer, because it has a good selectivity for wet etching between $\mathrm{A} \ell$ and dielectric materials such as $\mathrm{SiO}_{2}$ and $\mathrm{Ta}_{2} \mathrm{O}_{5}-\mathrm{SiO}_{2}$. According to (1), we need to form a long and thin beam to reduce the driving voltage. However, generally speaking, a long beam tends to bend easily by nonuniform internal stress distribution along the thickness. Although

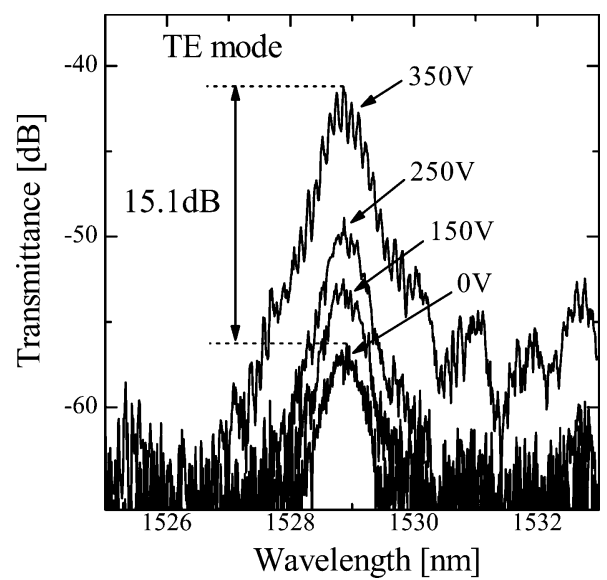

Fig. 4. Switching of drop-port response of MEMS-actuated microring resonator.

the thermal annealing process is commonly used to reduce the internal stress in the deposited film, the annealing process at high temperature was not applicable to the $\mathrm{A} \ell$ sacrifice layer, of which the melting point is $660^{\circ} \mathrm{C}$. To prevent the bending of beam, we deposited $\mathrm{SiO}_{2}$ upper cladding by plasma-enhanced chemical vapor deposition (PECVD) method, and etched back by about $20 \%$ of the surface layer, i.e., highly stressed portion, by reactive ion etching. Since the internal stress of films deposited by PECVD method is mainly localized in the layer close to the surface, the bending of beam can be prevented by etching back the surface of the deposited film [10]. As a result we successfully established the fabrication process of the straddle mounted beam with the beam length of $110 \mu \mathrm{m}$, and the initial beam deflection was reduced to be less than $0.1 \mu \mathrm{m}$ with the air gap of $0.65 \mu \mathrm{m}$. With this method, however, the beam length longer than $110 \mu \mathrm{m}$ turned out to be very difficult to make, since the resulting initial deflection became much greater than $0.1 \mu \mathrm{m}$.

On the other hand, the beam thickness should be thick enough to minimize the optical loss in the microring resonator, because a metal electrode is formed on the upper surface of the beam. From this limitation, the beam thickness was designed to be $3 \mu \mathrm{m}$, for which the absorption loss in the ring by the electrode was calculated to be less than $0.06 \mathrm{~dB} /$ round.

\section{EXPERIMENTAL RESULTS}

The switching characteristics of filter response of the MEMSactuated microring resonator were measured. The ring radius was $20 \mu \mathrm{m}$, beam length was $110 \mu \mathrm{m}$, and the initial air gap was $0.65 \mu \mathrm{m}$. The initial beam deflection was less than $0.1 \mu \mathrm{m}$. The measured results are shown in Figs. 4 and 5. The extinction ratio of drop port was $15.1 \mathrm{~dB}$ for TE polarization and $13.7 \mathrm{~dB}$ for TM polarization at the applied voltage of $350 \mathrm{~V}$. Since the waveguide width $(1.5 \mu \mathrm{m})$ was wider than the single-mode condition $(1.2 \mu \mathrm{m})$, the first higher order mode may be supported. The small peak at around $1532 \mathrm{~nm}$ may be caused by the higher order mode or the orthogonal polarization.

From these experimental results, the tuning range of efficiency and the beam deflection were evaluated in the following way. First, the initial air gap was assumed to be the designed 


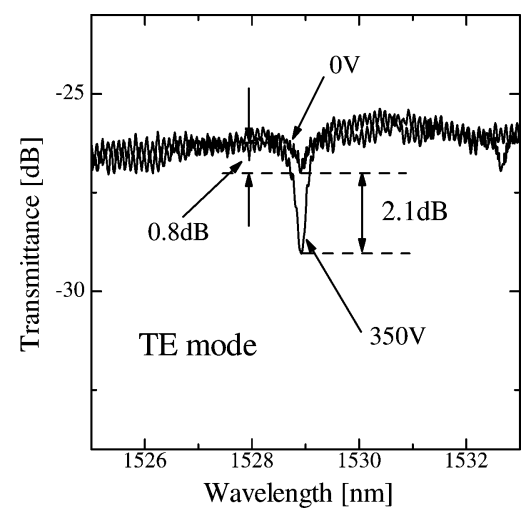

Fig. 5. Switching of through-port response of MEMS-actuated microring resonator.

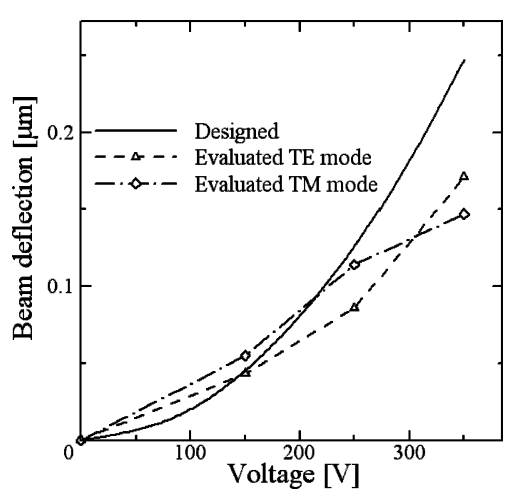

Fig. 6. Designed and evaluated beam deflection versus applied voltage.

value of $0.65 \mu \mathrm{m}$ in the OFF-state. Then, from Fig. 2, the coupling efficiency was evaluated to be 0.0016 for TE polarization and 0.00078 for TM polarization. By fitting the measured filter response of drop port in the OFF-state (Fig. 4) to the theoretical filter spectrum derived from this coupling efficiency in the OFF-state, the propagation loss in the ring resonator was evaluated to be $0.67 \mathrm{~dB} /$ round for TE polarization and $0.45 \mathrm{~dB} /$ round for TM polarization. Finally, the coupling efficiency in the ON-state was evaluated from the measured extinction ratio and the evaluated ring loss. The tuning range of coupling efficiency was evaluated to be from 0.0016 to 0.011 for TE polarization and from 0.00078 to 0.0042 for TM polarization, respectively, which corresponds to the beam deflection of $0.17 \mu \mathrm{m}$ for TE and $0.15 \mu \mathrm{m}$ for TM polarization. The maximum deflection was calculated from the measured data of filter responses for TE and TM mode. Fig. 6 shows the beam deflection versus applied voltage for designed and evaluated cases. The discrepancies between two cases are due to the experimental errors since the deflection should depend only on applied voltage.

At the applied voltage of $350 \mathrm{~V}$, the designed value of the beam deflection was $0.25 \mu \mathrm{m}$. The difference between designed and evaluated values seems to be caused by the residual internal stress of $\mathrm{SiO}_{2}$ upper cladding. The extinction ratio of through port was $2.1 \mathrm{~dB}$ for TE and $1.5 \mathrm{~dB}$ for TM polarization at the applied voltage of $350 \mathrm{~V}$. The loss in the OFF-state of the hitless WSS, which was previously reported, was $3 \mathrm{~dB}$ [7], and that of the device in this study was successfully reduced to $0.8 \mathrm{~dB}$.
Although the loss of $0.8 \mathrm{~dB}$ remained, this loss can be reduced by decreasing the coupling efficiency in the OFF-state. In the similar way, the device with the beam length of $100 \mu \mathrm{m}$ was measured and the extinction ratio of drop port was $8.1 \mathrm{~dB}$ for TE and TM polarizations.

Next the reproducibility of the filter response was measured. After the second cycle, the change in center wavelength was less than $0.1 \mathrm{~nm}$ within the measurement accuracy, and no degradation of the filter response was observed. A high reproducibility of the filter response was obtained, which implied that this device did not reach the sticking state. The switching time was measured to be $60 \mu \mathrm{s}$.

\section{CONCLUSION}

The coupling efficiency control of vertically coupled microring resonator filter was realized, and the extinction ratio of drop port response of $15.1 \mathrm{~dB}$ was demonstrated at the applied voltage of $350 \mathrm{~V}$. Different from the planar MEMS structure in which the busline and the microdisk resonator are formed in the same layer [11], the beam structure in our device can involve a microheater as the upper electrode. Therefore, this device is suitable for the simultaneous integration of MEMS switch and TO tunable filter, which can realize the perfect hitless wavelength switching.

\section{REFERENCES}

[1] S. C. Hagness, D. Rafizaden, S. T. Ho, and A. Taflove, "FDTD microcavity simulations: Design and experimental realization of waveguide-coupled single-mode ring and whispering-gallery-mode disk resonators," J. Lightw. Technol., vol. 15, no. 11, pp. 2154-2164, Nov. 1997.

[2] B. E. Little, S. T. Chu, H. A. Haus, J. Foresi, and J. P. Laine, "Microring resonator channel dropping filters," J. Lightw. Technol., vol. 15, no. 6, pp. 998-1005, Jun. 1997.

[3] S. T. Chu, B. E. Little, W. Pan, T. Kaneko, and Y. Kokubun, "An eight-channel add-drop filter using vertical coupled microring resonators over a cross grid," IEEE Photon. Technol. Lett., vol. 11, no. 6 , pp. 691-693, Jun. 1999.

[4] Y. Yanagase, S. Yamagata, and Y. Kokubun, "Wavelength tunable polymer microring resonator filter with $9.4 \mathrm{~nm}$ tuning range," Electron. Lett., vol. 39, no. 12, pp. 922-924, 2003.

[5] G. Sekiguchi, S. Yamagata, and Y. Kokubun, "Polarization-independent tuning of widely tunable vertically coupled microring resonator using thermo-optic effect," Jpn. J. Appl. Phys., vol. 44, no. 4A, pp. 1792-1796, 2005.

[6] S. Yamagata, T. Kato, and Y. Kokubun, "Non-blocling wavelength channel switch using TO effect of doubles series coupled microring resonator," Electron. Lett., vol. 41, no. 10, pp. 593-595, 2005.

[7] Y. Goebuchi, T. Kato, and Y. Kokubun, "Fast and stable wavelength selective switch using double-series coupled dielectric microring resonator," IEEE Photon. Technol. Lett., vol. 18, no. 3, pp. 538-540, Mar. 2006.

[8] T. Kato and Y. Kokubun, "Three-dimensional propagation analysis of coupling efficiency using segmentation and local normal mode expansion for vertically coupled microring resonator filter," J. Lightw. Technol., vol. 23, no. 8, pp. 2549-2554, Aug. 2005.

[9] Y. Kokubun, Y. Hatakeyama, M. Ogata, S. Suzuki, and N. Zaizen, "Fabrication technologies for vertically coupled microring resonator with multilevel crossing busline and ultracompact-ring radius," IEEE J. Sel. Topics Quantum Electron., vol. 11, no. 1, pp. 4-10, Jan./Feb. 2005.

[10] Y. Kageyama, "Analysis and control of stress distribution in polycrystalline silicon thin film," Central Inst. Toyota R\&D Rev., vol. 34, no. 1, pp. 3-9, 1999.

[11] M.-C. M. Lee and M. C. Wu, "MEMS-actuated microdisk resonators with variable power coupling ratios," IEEE Photon. Technol. Lett., vol. 17, no. 5, pp. 1034-1036, May 2005. 